\title{
Magnetic Dipole Excitation of an Insulated Conductor of Finite Length
}

\author{
DAVID A. HILL, FELLOW, IEEE
}

\begin{abstract}
Excitation of currents on an insulated conductor of finite length and with arbitrary terminations is studied for a magnetic dipole source. For matched terminations, the results agree closely with previous results for an infinitely long conductor, but other terminations produce end reflections that cause standing waves. Specific calculations are presented for a vertical magnetic dipole source, because this source produces the appropriate horizontal electric field and could be used in a borehole-to-borehole configuration. Numerical results for the induced current and secondary magnetic field indicate that long conductors produce a strong anomaly over a broad frequency range for any type of termination.
\end{abstract}

\section{INTRODUCTION}

$\mathrm{T}$ EXCITATION of currents on underground conductors is important in many applications. Power lines and rails in tunnels can enhance transmission for mine communications [1]. Electromagnetic probing of the Earth can be influenced by the presence of cables or pipes [2], [3]. Most work on dipole excitation of conductors has treated infinitely long conductors [4], [5]. This idealization allows a spatial Fourier transform formulation that simplifies the calculation of the current distribution.

In this paper, our model consists of an insulated conductor of finite length in a homogeneous, lossy Earth, excited by a vertical magnetic dipole. The insulated conductor has an arbitrary terminating impedance at each end. The theory of excitation of an insulated conductor by an external field has been given by King [6], and we use it for the particular case of magnetic dipole excitation. One application of this case is the detection of long conductors in tunnels by means of vertical transmitting and receiving magnetic dipoles in vertical boreholes. This application was studied in [5] for infinitely long conductors, and in this paper we study the effects of finite conductor length.

\section{Magnetic Dipole Fields}

The geometry for the magnetic dipole and conductor is shown in Fig. 1. The conductor of length $2 h$ extends from $-h$ to $h$ on the $z$ axis. A vertical ( $y$ directed) magnetic dipole of magnetic moment $I A$ is located at $\left(x_{d}, y_{d}, z_{d}\right)$. We choose a vertical magnetic dipole because it radiates

Manuscript received June 8, 1989; revised January 2, 1990. This work was supported by the U.S. Army Belvoir RD\&E Center.

The author is with the Electromagnetic Fields Division, National Institute of Standards and Technology, Boulder, CO 80303.

IEEE Log Number 9034313.

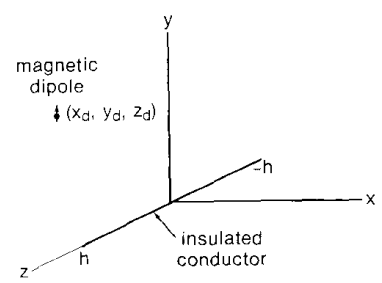

Fig. 1. Geometry for a magnetic dipole source and an insulated conductor of length $2 h$.

a horizontal electric field which excites axial ( $z$ directed) currents in the conductor.

The fields due to the magnetic dipole in the absence of the conductor can be derived from a magnetic Hertz vector with only a $y$ component $\Pi_{y}^{d *}$. For $\exp (j \omega t)$ time dependence, $\Pi_{y}^{d *}$ is [5]

$$
\Pi_{y}^{d *}=\frac{I A e^{-j k r_{d}}}{4 \pi r_{d}}
$$

where $r_{d}=\left[\left(x-x_{d}\right)^{2}+\left(y-y_{d}\right)^{2}+\left(z-z_{d}\right)^{2}\right]^{1 / 2}$, $k=\omega[\mu(\epsilon-j \sigma / \omega)]^{1 / 2}$, and $\mu, \epsilon$, and $\sigma$ are the earth permeability, permittivity, and conductivity. The electric and magnetic fields $E^{d}$ and $H^{d}$ due to the magnetic dipole are [7]

$$
\begin{aligned}
\boldsymbol{E}^{d} & =-j \omega \mu \nabla \times\left(\boldsymbol{e}_{y} \Pi_{y}^{d *}\right) \\
\boldsymbol{H}^{d} & =\left(\nabla \nabla \cdot+k^{2}\right)\left(\boldsymbol{e}_{y} \Pi_{y}^{d *}\right)
\end{aligned}
$$

where $\boldsymbol{e}_{y}$ is a unit vector in the $y$ direction.

For reception with a second vertical magnetic dipole, the $y$ component $H_{y}^{d}$ of the magnetic field is required. This is obtained from substituting (1) into (2) and carrying out the differentations:

$$
\begin{aligned}
H_{y}^{d}= & \frac{I A e^{-j k r_{d}}}{4 \pi r_{d}}\left\{k^{2}\left[1-\frac{\left(y-y_{d}\right)^{2}}{r_{d}^{2}}\right]+\frac{\left(j k r_{d}+1\right)}{r_{d}^{2}}\right. \\
& \left.\cdot\left[\frac{3\left(y-y_{d}\right)^{2}}{r_{d}^{2}}-1\right]\right\} .
\end{aligned}
$$

For excitation of the conductor, the $z$ component $E_{z}^{d}$ is required, and it is also obtained from (1) and (2):

$$
E_{z}^{d}=j \omega \mu \frac{I A e^{-j k r_{d}}}{4 \pi r_{d}} \frac{\left(x-x_{d}\right)}{r_{d}}\left[j k+\frac{1}{r_{d}}\right] .
$$

U.S. Government work not protected by U.S. copyright 


\section{Conductor Characteristics}

A model for a buried insulated conductor is shown in Fig. 2. It consists of a metal conductor of radius $a$ surrounded by an insulating region of outer radius $b$. The metal has conductivity $\sigma_{m}$ and magnetic permeability $\mu_{m}$, and the insulation has permittivity $\epsilon_{i}$ and free-space permeability $\mu_{0}$.

For sufficiently low frequencies, the insulated conductor can be characterized by transmission line parameters. The series impedance per unit length $z_{L}$ is [6], [8]

$$
z_{L}=z_{1}^{i}+z_{3}^{i}+z^{e}
$$

where

$$
\begin{gathered}
z_{1}^{i}=\frac{\left(j \omega \mu_{m} / \sigma_{m}\right)^{1 / 2}}{2 \pi a}, \quad z_{3}^{i}=\frac{j \omega \mu}{2 \pi} \frac{H_{0}^{(2)}(k b)}{k b H_{1}^{(2)}(k b)}, \\
z^{e}=\frac{j \omega \mu_{0}}{2 \pi} \ln (b / a)
\end{gathered}
$$

and $H_{0}^{(2)}$ and $H_{1}^{(2)}$ are Hankel functions of the second kind [9]. In (5), $z_{1}^{i}$ represents the effect of finite conductivity of the inner conductor, $z_{3}^{i}$ represents the contribution to the impedance from the fields in the Earth, and $z^{e}$ represents the contribution from the fields in the insulation region. In (5) and throughout this paper, we take the complex conjugate of King's expressions because he used exp $(-i \omega t)$ time dependence. The shunt admittance per unit length $y_{L}$ is [6], [8]

$$
y_{L}=\frac{j \omega 2 \pi \epsilon_{i}}{\ln (b / a)} .
$$

The characteristic impedance $Z_{c a}$ and the wave number $k_{L}$ are [6], [8]

$$
Z_{c a}=\left(z_{L} / y_{L}\right)^{1 / 2}
$$

and

$$
k_{L}=\left(-z_{L} y_{L}\right)^{1 / 2} \text {. }
$$

The main requirements for the validity of (5)-(7) are that the wavenumber of the Earth be large compared to that of the insulation $\left(\left|k^{2}\right| \gg \omega^{2} \mu_{0} \epsilon_{i}\right)$ and that the insulated conductor be electrically thin $(|k| b<<1)$.

To obtain a numerical check of the transmission line approximations in (5)-(7), we compared the real and imaginary parts of the wavenumber $k_{L}=\beta_{L}-j \alpha_{L}$ in (7) with the solution of the more rigorous mode equation described in [5] for the following parameters: $a=0.5 \mathrm{~cm}$, $\sigma_{m}=5.7 \times 10^{7} \mathrm{~S} / \mathrm{m}, \epsilon_{i} / \epsilon_{0}=1, \epsilon / \epsilon_{0}=10, \sigma=5 \times$ $10^{-3} \mathrm{~S} / \mathrm{m}$, and $\mu_{m} / \mu_{0}=\mu / \mu_{0}=1$. These parameters also apply to all the following figures. The Earth parameters are typical of those for nonmagnetic rock. Since we have set $\epsilon_{i}=\epsilon_{0}$, we can think of $b-a$ as the distance of the conductor from the wall of an air-filled tunnel. The conductivity $\sigma_{m}$ is that of copper, and the results are only weakly dependent on the conductor radius $a$. Numerical results for the attenuation rate $\left(=8.686 \alpha_{L}\right.$ in $\left.\mathrm{dB} / \mathrm{m}\right)$ are shown in Fig. 3 as a function of frequency for several

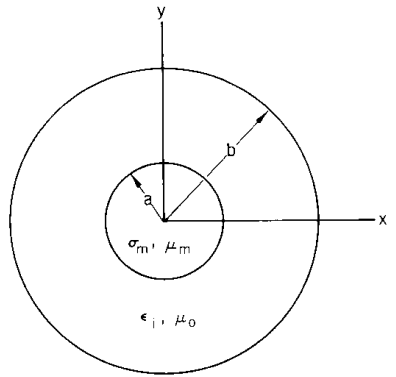

Fig. 2. Geometry for an insulated conductor.

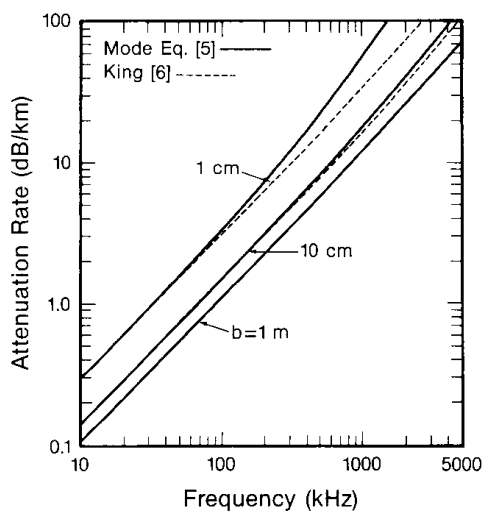

Fig. 3. Attenuation rate for an insulated conductor.

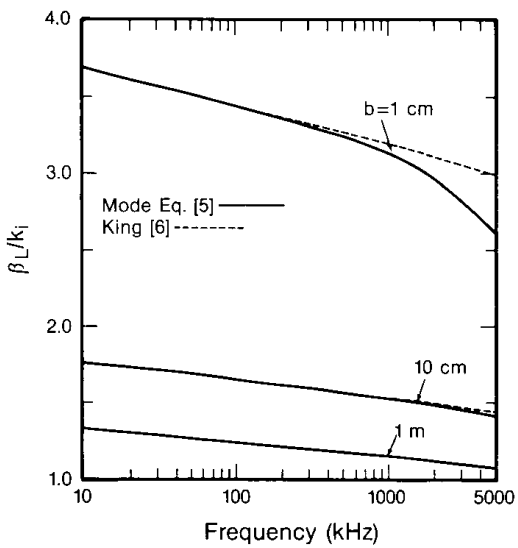

Fig. 4. Normalized phase constant for an insulated conductor.

values of $b$. As expected, the attenuation rate increases with frequency, and the agreement with the mode equation is best for low frequencies. Numerical results for the phase constant $\beta_{L}$ are shown in Fig. 4 , and $\beta_{L}$ is normalized to the wavenumber of the insulation, $k_{i}=$ $\omega\left(\mu_{0} e_{i}\right)^{1 / 2}$. Again, the agreement is best for low frequencies. The characteristic impedance $Z_{c a}$ is important in determining the induced current, and numerical results from (7) are shown in Fig. 5. 


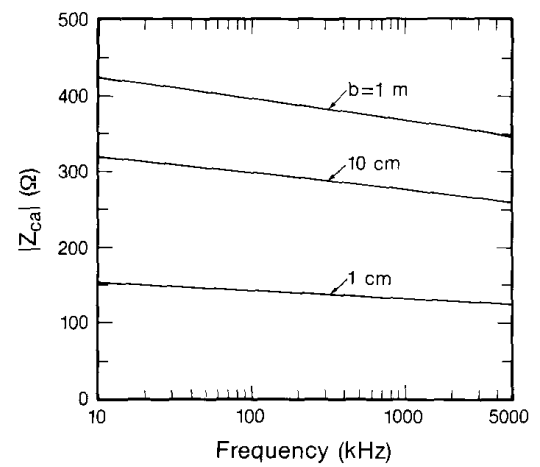

Fig. 5. Characteristic impedance of an insulated conductor.

\section{INDUCED CURRENT}

King [6] has presented expressions for the current in an insulated conductor of finite length and with arbitrary terminations when the incident field is either a gap feed (antenna problem) or an incident field (scattering problem). Numerous comparisons of the theoretical and measured current distributions for a gap feed are given in [10], and the agreement is generally good. For incident field excitation, the induced current is written as an integral of the incident field times the Green's function over the length of the conductor, and King [6] has evaluated this integral for uniform plane-wave incidence. For magnetic dipole excitation, the integral expression for the induced current $I\left(z^{\prime}\right)$ is written in the following form:

$$
I\left(z^{\prime}\right)=\int_{-h}^{h} E_{z}^{d}\left(z_{s}\right) G_{t}\left(z^{\prime}, z_{s}\right) d z_{s}
$$

where $E_{z}^{d}\left(\mathrm{z}_{s}\right)$ is obtained from (4) and evaluated at $z=$ $z_{s}$ on the $z$ axis. The Green's function $G_{t}$ for a terminated transmission line is obtained by writing King's result [6], [11] in terms of circular functions:

$$
G_{i}\left(z^{\prime}, z_{s}\right)= \begin{cases}\frac{j}{Z_{c a}} \frac{\sin \left[k_{L}\left(h+z_{s}\right)-j \theta_{-}\right] \sin \left[k_{L}\left(h-z^{\prime}\right)-j \theta_{-}\right]}{\sin \left(2 k_{L} h-j \theta_{-}-j \theta_{+}\right)}, & z^{\prime} \geq z_{s} \\ \frac{j}{Z_{c a}} \frac{\sin \left[k_{L}\left(h+z^{\prime}\right)-j \theta_{-}\right] \sin \left[k_{L}\left(h-z_{s}\right)-j \theta_{+}\right]}{\sin \left(2 k_{L} h-j \theta_{-}-j \theta_{+}\right)}, & z^{\prime} \leq z_{s}\end{cases}
$$

where

$$
\theta_{-}=\operatorname{coth}^{-1}\left(Z_{-} / Z_{c a}\right) \text { and } \theta_{+}=\operatorname{coth}^{-1}\left(Z_{+} / Z_{c a}\right)
$$

In (10), $Z_{-}$and $Z_{+}$are the impedances terminating the insulated conductor at $-h$ and $+h$.

For the special cases of open ends $\left(Z_{-}=Z_{+}=\infty\right)$, short-circuited ends $\left(Z_{-}=Z_{+}=0\right)$, and matched ends $\left(Z_{-}=Z_{+}=Z_{c a}\right.$ ), the results in (9) and (10) simplify to end is often encountered in practice where the actual conductor is suspended and no grounding is attempted [12]. The opposite case of a short circuit represents perfect grounding of the conductor to the surrounding rock. Fairly the results given in the Appendix. The case of an open good grounding can sometimes be achieved with roof bolts or some other grounding conductor if the grounding conductor is not corroded and makes good contact with the rock. The case of a matched termination is highly idealized, but this reflectionless case is useful for comparison with the earlier calculations for infinitely long conductors [5].

A computer program to evaluate the current distribution in (8) by numerical integration was written. Fig. 6 shows the effect of the conductor termination for the following parameters: $I A=1 \mathrm{~A} \cdot \mathrm{m}^{2}, x_{d}=-15 \mathrm{~m}, y_{d}=0, z_{d}=$ $250 \mathrm{~m}, 2 h=2 \mathrm{~km}$, and $f=100 \mathrm{kHz}$. The matched line shows a smooth decay of the current with no end reflections, and the result for an infinitely long line [5] is also shown for comparison. This is a good check of the numerical integration, because the result in [5] was obtained by a totally different Fourier transform method. Additional comparisons between the matched line and the infinitely long line showed good agreement for frequencies of less than about $500 \mathrm{kHz}$. Another requirement of this transmission line model is that the insulation thickness $(b-a)$ be sufficiently thick so that $\left|k_{L}^{2}\right| \ll<K^{2} \mid$ and that the propagation in the insulation dominates the propagation in the surrounding medium. Also, both the magnetic dipole source and the observation point must be located at least several radii $b$ from the conductor so that only the rotationally symmetric current is significant.

In Fig. 6, the end reflections for the open and shorted lines are very prominent and the current for the open goes to zero at the ends. When the source is located near one end $\left(z_{d}=h\right)$, the open line is difficult to excite, as shown in Fig. 7. Here we set $z_{d}=h=1 \mathrm{~km}$, and the other parameters remain the same. The matched and shorted lines are strongly excited, but the open line is only weakly excited.
Because the open line is often encountered in practice [12], we study the effect of source location on the excitation of an open line in Fig. 8: The excitation improves as the source is moved away from the end toward the center of the line, and this is consistent with experimental results [12]. From Fig. 4 we can calculate that the effective wavelength of the line $\left(2 \pi / \beta_{L}\right)$ is approximately 940 $\mathrm{m}$ at $100 \mathrm{kHz}$. When the source is approximately a quarter wavelength $(235 \mathrm{~m})$ from the end of the line, then the reflected wave is in phase with the direct wave and the excitation is very strong. The situation in Fig. 8 is actually more complicated because of multiple reflections from both ends. 


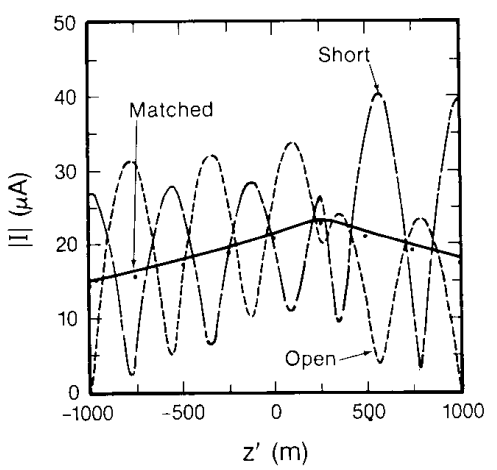

Fig. 6. Current induced on an insulated conductor by a magnetic dipole source. The result $(\cdots)$ for an infinitely long conductor $[5\}$ is also shown.

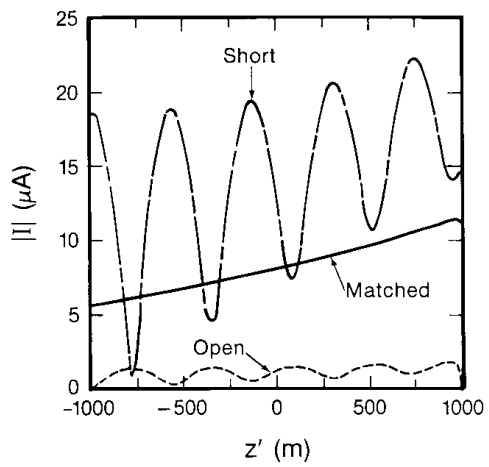

Fig. 7. Current induced on an insulated conductor by a magnetic dipole located near the end $\left(z_{d}=h\right)$.

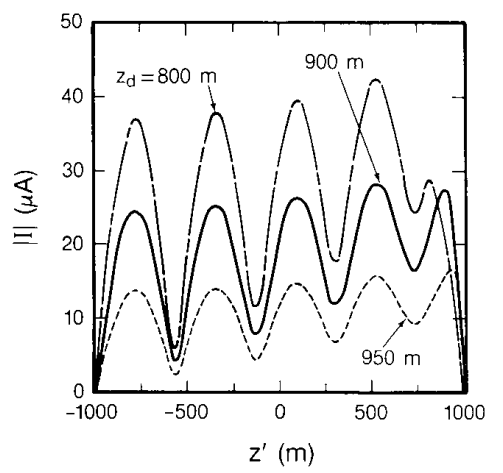

Fig. 8. Current induced on an open insulated conductor.

In Fig. 9 we show the effect of moving the source farther away from the line for the case of a shorter line $(2 h$ $=1 \mathrm{~km}$ ). Again, $z_{d}=250 \mathrm{~m}$. The induced current falls off rapidly with increasing $\left|x_{d}\right|$ because of the exponential attenuation of the incident field in the lossy Earth.

In Fig. 10 we show the effect of frequency on the induced current for an open line of length $2 h=1 \mathrm{~km}$. Again, $z_{d}=250 \mathrm{~m}$ and $x_{d}=-15 \mathrm{~m}$. Rapid oscillations occur at $500 \mathrm{kHz}$ because of the shorter wavelength. The

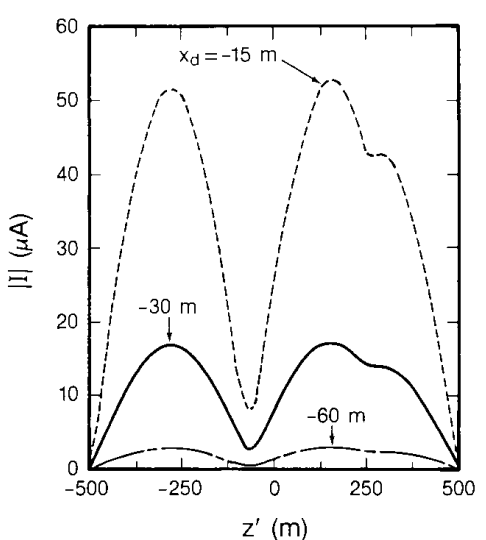

Fig. 9. Current induced on an open insulated conductor for various dipole distances.

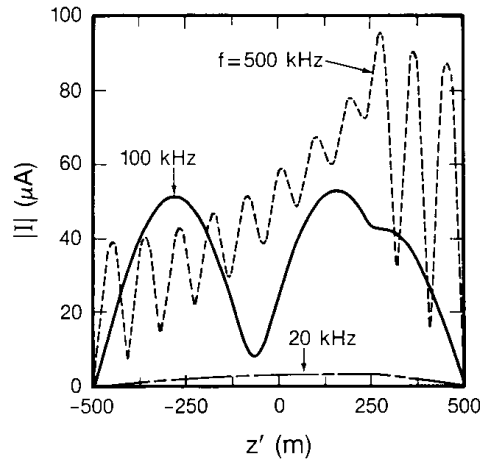

Fig. 10. Current induced on an open insulated conductor for various frequencies.

excitation is poor at $\angle 0 \mathrm{kHz}$ because the line is electrically short (about one-quarter wavelength). To achieve strong excitation, the frequency should be high enough so that the conductor length $2 h$ is at least a half wavelength. Some experimental results for magnetic dipole excitation at frequencies from 50 to $500 \mathrm{kHz}$ have been obtained for open wires, and the agreement of the current distribution and propagation constant with theoretical results is generally good [12].

\section{Magnetic Field}

The primary fields of the magnetic dipole are given by (2). The fields of the currents induced in the conductor can be derived from an electric Hertz vector with only a $z$ component $\Pi_{z}^{c}[5]$ :

$$
\Pi_{z}^{c}=\frac{1}{4 \pi(\sigma+j \omega \epsilon)} \int_{-h}^{h} \frac{I\left(z^{\prime}\right) e^{-j k r_{c}}}{r_{c}} d z^{\prime}
$$

where

$$
r_{c}=\left[x^{2}+y^{2}+\left(z-z^{\prime}\right)^{2}\right]^{1 / 2} .
$$




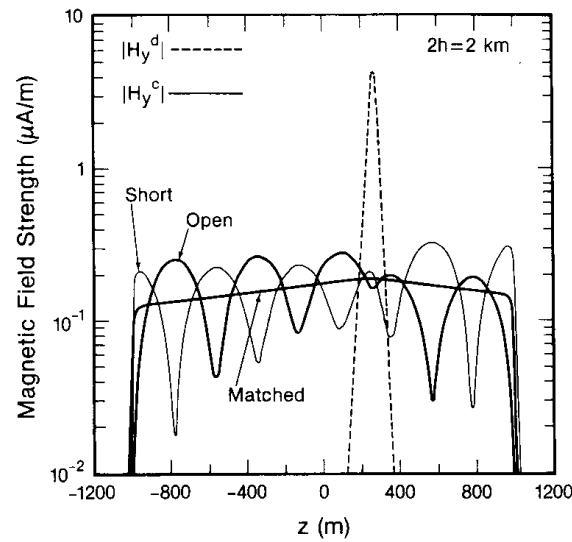

Fig. 11. Primary and secondary vertical magnetic field for magnetic field excitation.

The electric and magnetic fields $\boldsymbol{E}^{c}$ and $\boldsymbol{H}^{c}$ due to the conductor are [5]

$$
\boldsymbol{E}^{c}=\left(\nabla \nabla \cdot+k^{2}\right)\left(\boldsymbol{e}_{z} \Pi_{z}^{c}\right)
$$

and

$$
\boldsymbol{H}^{c}=(\sigma+j \omega \epsilon) \nabla \times\left(\boldsymbol{e}_{z} \Pi_{z}^{c}\right) .
$$

For reception with a vertical magnetic dipole, the $y$ component $H_{y}^{c}$ of the magnetic field is required, and this is obtained from (11) and (12):

$$
H_{y}^{c}=\int_{-h}^{h} I\left(z^{\prime}\right) G_{y}^{c} d z^{\prime}
$$

where

$$
G_{y}^{c}=\left(j k+\frac{1}{r_{c}}\right) \frac{x e^{-j k r_{c}}}{4 \pi r_{c}^{2}} .
$$

A computer program was written to evaluate (13) by numerical integration. In Fig. 11 we show the primary and secondary magnetic fields observed at $x=15 \mathrm{~m}$ and $y=0$ for three different terminations. The remaining parameters are the same as those in Fig. 6. The primary magnetic field decays rapidly in $z$, and the secondary magnetic field closely follows the current distributions in Fig. 6. For a matched termination, the secondary magnetic field agrees closely with that of the infinitely long conductor in [5]. For all terminations the magnetic field drops off rapidly beyond the end of the conductor $(|z|>$ $1 \mathrm{~km})$. Because the secondary magnetic field dominates the primary magnetic field except near the source, the conductor should be easy to detect when both the transmitter and receiver are located near the conductor.

The frequency dependence of the magnetic field for an open line is shown in Fig. 12. The secondary magnetic fields closely follow the current distributions shown in Fig. 10. A frequency of $20 \mathrm{kHz}$ is too low to produce a strong secondary magnetic field because the conductor is electrically short. Additional calculations at other frequencies show that the broad frequency range from about

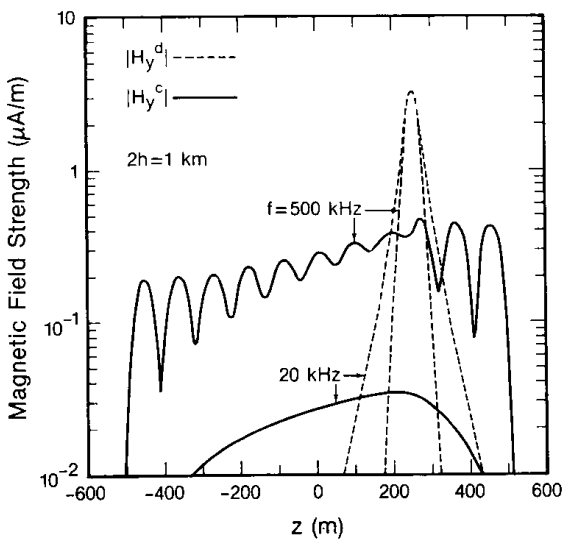

Fig. 12. Primary and secondary vertical magnetic field for two frequencies.

50 to $500 \mathrm{kHz}$ should be useful in detecting long conductors.

\section{ConClusions}

The results presented here indicate that a vertical magnetic dipole can excite significant axial currents on insulated conductors of finite length. The current distribution depends strongly on the terminations, and the results for a matched termination agree closely with previous results for infinitely long conductors [5]. For open terminations, the source should be somewhat removed from the ends to excite the conductor effectively. Also, the frequency should be high enough so that the conductor is at least one-half wavelength long. When the conductor is effectively excited, the secondary field dominates the primary field except near the source. Thus the detection of conductors appears feasible when both the transmitting and receiving antennas are located fairly close to the conductor. A frequency range from about 50 to $500 \mathrm{kHz}$ is most promising.

A number of extensions to this work would be useful. Excitation (or reception) by electric or magnetic dipoles of other orientations or by long electric line sources could be examined. The presence of the air-Earth interface could be included for cases of shallow conductor depth. Although the insulated conductor of finite length considered here is an improvement over the infinitely long conductor model [5], multiple conductors or grounded conductors of finite length [13] could also be considered. Grounded conductors could represent rails, and the imperfect contact between rail segments or with the tunnel rock could also be considered. The conductor terminations could be studied in more detail and the end capacitance of open lines [14] could be taken into account. In this paper we displayed only the magnitude of the induced current and magnetic field, but the phase might also contain useful information. Also, Earth layering or inhomogeneities could be studied to obtain an estimate of the competing geologic noise [15]-[17]. 


\section{APPENDIX \\ SPECial Terminations}

For open ends $\left(Z_{-}=Z_{+}=\infty\right)$, we have $\theta_{-}=\theta_{+}=$ 0 . In this case, $(9)$ reduces to

$G_{t}\left(z^{\prime}, z_{s}\right)=\left\{\begin{array}{c}\frac{j}{Z_{c a}} \frac{\sin \left[k_{L}\left(h+z_{s}\right)\right] \sin \left[k_{L}\left(h-z^{\prime}\right)\right]}{\sin \left(2 k_{L} h\right)}, \\ z^{\prime} \geq z_{s} \\ \frac{j}{Z_{c a}} \frac{\sin \left[k_{L}\left(h+z^{\prime}\right)\right] \sin \left[k_{L}\left(h-z_{s}\right)\right]}{\sin \left(2 k_{L} h\right)} \\ z^{\prime} \leq z_{s} .\end{array}\right.$

For short-circuited ends $\left(Z_{-}=Z_{+}=0\right)$, we have $\theta_{-}$ $=\theta_{+}=j \pi / 2$. In this case, (9) reduces to

$G_{t}\left(z^{\prime}, z_{s}\right)=\left\{\begin{array}{c}\frac{-j}{Z_{c a}} \frac{\cos \left[k_{L}\left(h+z_{s}\right)\right] \cos \left[k_{L}\left(h-z^{\prime}\right)\right]}{\sin \left(2 k_{L} h\right)}, \\ z^{\prime} \geq z_{s} \\ \frac{-j}{Z_{c a}} \frac{\cos \left[k_{L}\left(h+z^{\prime}\right)\right] \cos \left[k_{L}\left(h-z_{s}\right)\right]}{\sin \left(2 k_{L} h\right)} \\ z^{\prime} \leq z_{s} .\end{array}\right.$

For matched ends $\left(Z_{-}=Z_{+}=Z_{c a}\right)$, we have $\theta_{-}=\theta_{+}$ $=\infty$. In this case, $(9)$ reduces to

$$
G_{t}\left(z^{\prime}, z_{s}\right)=\frac{e^{-j k_{L}\left|z^{\prime}-z_{s}\right|}}{2 Z_{c a}} .
$$

\section{ACKNOWLEDGMENT}

The author would like to thank Dr. L. G. Stolarczyk and Dr. F. Ruskey for helpful discussions.

\section{REFERENCES}

[1] J. R. Wait and D. A. Hill, "Radio frequency transmission via a trolley wire in a tunnel with rail return," IEEE Trans. Antennas Propagat., vol. AP-25, pp. 248-253, 1977.

[2] J. R. Wait and K. R. Umashankar, "Analysis of the earth resistivity response of buried cables," Pure Appl. Geophys., vol. 117, pp. 711 $742,1978$.

[3] K. Tsubota and J. R. Wait, "The frequency and the time-domain responses of a buried axial conductor," Geophysics, vol. 45, pp. 941$951,1980$.
[4] D. A. Hill and J. R. Wait, "Coupling between a dipole antenna and an infinite cable over an ideal ground plane," Radio $S_{c i}$, vol. 12 , pp. 231-238, 1977.

[5] D. A. Hill, "Magnetic dipole excitation of a long conductor in a lossy medium,"' IEEE Trans. Geosci. Remote Sensing, vol. 26, pp. 720$725,1988$.

[6] R. W. P. King. "The insulated conductor as a scattering antenna in a relatively dense medium," IEEE Trans. Antennas Propagat., vol. AP-24, pp. 327-330, 1976.

[7] J. A. Stratton, Electromagnetic Theory. New York: McGraw-Hill, 1941.

[8] T, T. Wu, R. W. P. King, and D. V. Giri, "The insulated dipole antenna in a relatively dense medium,"' Radio $\mathrm{Sci}$., vol. 8, pp. 699$709,1973$.

[9] M. Abramowitz and I. A. Stegun, Handbook of Mathematical Functions. Washington, DC: Nat. Bureau of Standards, 1964.

[10] R. W. P. King and G. S. Smith, Antennas in Matter. Cambridge, MA: MIT Press, 1981.

[11] R. W. P. King, Transmission Line Theory. New York: Dover, 1965.

[12] L. G. Stolarczyk, private communication, 1989.

[13] R. W. P. King, P. F. Sforza, and T. I. S. Boak, III, "The current in a parasitic antenna in a dissipative medium," IEEE Trans. Antennas Propagat., vol. AP-22, pp. 809-814, 1974

[14] W. C. Daywitt, "Complex admittance of a lossy coaxial open circuit with a hollow center conductor," Metrologia, vol. 24, pp. 13-22, 1987.

[15] P. A. Eaton and G. W. Hohmann, "An evaluation of electromagnetic methods in the presence of geologic noise," Geophysics, vol. 52, pp. $1106-1126,1987$.

[16] R. Greenfield, "Borehole radar clutter," presented at the Nat. Radio Sci. Meet., Boulder, CO, January 4-6, 1989.

[17] D. A. Hill, "Clutter models for subsurface electromagnetic applications," Nat. Inst. Stand. and Tech., Boulder, CO, Rep. NISTIR 89$3909,1989$.

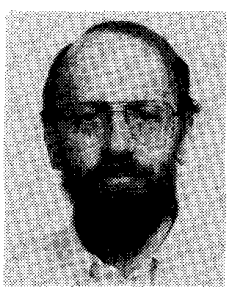

David A. Hill (M'72-SM'76-F'87) was born in Cleveland, $\mathrm{OH}$, on April 21, 1942. He received the B.S.E.E. and M.S.E.E. degrees from Ohio University, Athens, in 1964 and 1966, respectively, and the Ph.D. degree in electrical engineering from Ohio State University, Columbus, in 1970 .

Since 1970 he has been a member of the Boulder scientific community. From 1970 to 1971 he was a Visiting Fellow with the Cooperative Institute for Research in Environmental Sciences, where he worked on pulse propagation. From 1971 to 1982 he was with the Institute for Telecommunication Sciences, where he worked on theoretical problems in antennas and propagation. Since 1982 he has been in the Electromagnetic Fields Division of the National Institute of Standards and Technology, where he has been working on EMC/EMI problems. He is also a Professor Adjoint in the Department of Electrical and Computer Engineering of the University of Colorado.

Dr. Hill is a member of URSI Commissions B, E, and F. He has served as an Associate Editor for the IEEE TRANSACTIONS ON GEOSCIENCE AND Remote Sensing and the IEEE Transactions on Antennas and PropaGATION. 